\title{
Increased cortical inhibition deficits in first-episode schizophrenia with comorbid cannabis abuse
}

\author{
Thomas Wobrock • Alkomiet Hasan • Berend Malchow • Claus Wolff-Menzler • \\ Birgit Guse • Nicolas Lang • Thomas Schneider-Axmann • Ullrich K. H. Ecker • \\ Peter Falkai
}

Received: 9 July 2009 / Accepted: 16 November 2009 / Published online: 9 December 2009

(C) The Author(s) 2009. This article is published with open access at Springerlink.com

\begin{abstract}
Rationale/objectives There is a high prevalence of substance use disorder (SUD) in first-episode schizophrenia (SZ), but its contribution to the underlying SZ pathophysiology remains unclear. Several studies using transcranial magnetic stimulation (TMS) have observed abnormalities in human motor cortex (M1) excitability in SZ. Studies on cortical excitability comparing SZ patients with and without comorbid substance abuse are lacking.

Methods A total of 29 first-episode SZ patients participated in this study; 12 had a history of comorbid cannabis abuse (SZ-SUD) and 17 did not (SZ-NSUD). We applied TMS to right and left $\mathrm{M} 1$ areas to assess the resting motor threshold (RMT), short-interval cortical inhibition (SICI), intracortical facilitation (ICF), and the contralateral cortical silent period (CSP).

Results In SICI and ICF conditions, right M1 stimulation led to significantly higher motor evoked potential ratios in SZ-SUD compared to SZ-NSUD. This suggests lower cortical inhibition and increased ICF in first-episode SZ
\end{abstract}

T. Wobrock and A. Hasan contributed equally.

T. Wobrock $(\bowtie) \cdot$ A. Hasan · B. Malchow $\cdot$ C. Wolff-Menzler •

B. Guse $\cdot$ T. Schneider-Axmann $\cdot$ P. Falkai

Department of Psychiatry and Psychotherapy,

Georg-August-University Göttingen,

Von-Siebold-Strasse 5,

37075 Göttingen, Germany

e-mail: twobroc@gwdg.de

N. Lang

Department of Neurology, Christian-Albrechts-University Kiel, 24195 Kiel, Germany

U. K. H. Ecker

School of Psychology, University of Western Australia,

Crawley, WA 6009, Australia with previous cannabis abuse. There were no group differences in RMT and CSP duration. Neither were there any significant correlations between psychopathology (as indexed by Positive and Negative Syndrome Scale), disease characteristics, the extent of cannabis abuse, and TMS parameters (SICI, ICF, and CSP).

Conclusions Comorbid cannabis abuse may potentiate the reduced intracortical inhibition and enhanced ICF observed in first-episode SZ patients in some previous studies. This finding suggests an increased alteration of $\mathrm{GABA}_{\mathrm{A}}$ and NMDA receptor activity in cannabis-abusing first-episode patients as compared to schizophrenia patients with no history of substance abuse. This may constitute a distinct vulnerability factor in this special population.

Keywords Schizophrenia · Substance abuse ·

Cortical inhibition - Transcranial magnetic stimulation

(TMS) $\cdot$ Cannabinoids $\cdot$ Electrophysiology

\section{Introduction}

Substance abuse is common in patients with schizophrenia. Various studies have reported prevalence rates for this psychiatric comorbidity ranging from $15 \%$ to $65 \%$, with nicotine, alcohol, and cannabis as the most frequently consumed substances (Cantor-Graae et al. 2001; Mueser et al. 1990; Wobrock and Soyka 2008). In particular, crosssectional studies have found that cannabis abuse is associated with an earlier onset of schizophrenia, and longitudinal birth cohort studies have suggested that cannabis abuse is an independent risk factor for the development of schizophrenia (Semple et al. 2005; Wobrock et al. 2007a). 
Although it has been demonstrated that cannabis can induce dopamine release (Voruganti et al. 2001) - and increased dopaminergic transmission is seen in schizophrenia (Abi-Dargham et al. 2000) - the understanding of the effects of cannabis consumption on the pathophysiology of schizophrenia and the neurobiological interaction between these two conditions is far from complete (Rathbone et al. 2008). In recent years, some evidence has accrued that alterations in the endogenous cannabinoid system are linked to schizophrenia. First, an association between a cannabinoid 1 receptor (CB1R) polymorphism and hebephrenic schizophrenia was observed (Ujike et al. 2002); second, the expression of CB1Rs in the brain of schizophrenia patients was found to be abnormal (Dean et al. 2001; Eggan et al. 2008); and third, the level of endocannabinoid anandamide was significantly elevated in the cerebrospinal fluid of antipsychotic-naive first-episode paranoid-type schizophrenia patients relative to healthy controls, affective disorder, and dementia patients (Giuffrida et al. 2004).

Animal studies have provided evidence for a link between GABAergic transmission and the cannabinoid system. Exogenous and endogenous cannabinoids take effect via CB1Rs and may influence GABAergic transmission in various cortical and subcortical brain areas (Hoffman and Lupica 2000, 2001; Katona et al. 1999, 2000).

Fittingly, some neurophysiological studies have reported disturbances of GABAergic transmission in schizophrenia patients. These studies have used paired-pulse transcranial magnetic stimulation (TMS; Kujirai et al. 1993) to assess mechanisms of Short-interval intracortical inhibition (SICI) and intracortical facilitation (ICF). There is strong evidence that SICI is mediated by GABAergic interneurons via $\mathrm{GABA}_{\mathrm{A}}$ receptors, and that ICF results from prevailing glutamatergic and weakened GABAergic interneuronal influence (Ziemann 2004). For the detection of $\mathrm{GABA}_{\mathrm{B}^{-}}$ mediated cortical inhibition, the TMS measurement of the contralateral cortical silent period (CSP) seems to be a useful tool (Siebner et al. 1998; Werhahn et al. 1999). Despite some controversy, a number of TMS studies have linked schizophrenia to distinct abnormalities of cortical excitability in the motoneural system. This research is mainly pointing toward a GABAergic dysfunction in schizophrenia (Daskalakis et al. 2002; Eichhammer et al. 2004; Fitzgerald et al. 2002a,b,c; Pascual-Leone et al. 2002; Wobrock et al. 2008). Another important study has found GABA-related transcripts in four cortical areas (dorsolateral prefrontal cortex, anterior cingulate cortex, and primary motor and primary visual cortices) to be lower in subjects with schizophrenia (Hashimoto et al. 2008).

However, beyond this, the neurophysiological impact of comorbid cannabis abuse in patients with schizophrenia is largely unknown. Various studies have revealed a reduction of event-related potentials - such as the auditory evoked
P300 - both in schizophrenia, including its prodrominal states, and after cannabis use (Coburn et al. 1998; Frommann et al. 2008; Ozgürdal et al. 2008; Roser et al. 2008). These findings can be taken to reflect reduced inhibition in cortico-subcortical loops, which in turn is suggestive of GABAergic dysfunction.

In the present study, first-episode schizophrenia patients with minimal exposure to antipsychotics were assessed with single and paired-pulse TMS measuring SICI, ICF, and CSP. While reduced SICI has already been demonstrated in this patient sample relative to healthy control subjects (Wobrock et al. 2008), the aim of the present study was to compare motoneural cortical excitability between firstepisode schizophrenia patients with and without a history of cannabis abuse. One recently published study found a reduction of SICI in chronic cannabis abusers that might be related to disturbances in $\mathrm{GABA}_{\mathrm{A}}$ transmission (Fitzgerald et al. 2009). The literature discussed above likewise suggests an alteration of GABAergic neurotransmission in cannabis-abusing subjects. We therefore hypothesized that comorbid schizophrenia patients would present with pronounced disturbances in cortical inhibition relative to patients without accompanying substance (cannabis) use disorder (SUD).

\section{Methods}

Subjects

Twenty-nine patients with first-episode schizophrenia (all paranoid subtype) participated in the study. They were recruited from the Saarland University Hospital between 2003 and 2006. Exclusion criteria included a history of dementia, neurological illness, severe brain injury or brain tumors, abuse of substances other than cannabis, including alcohol, or other contraindication to TMS. Each subject underwent a detailed biographic interview (Bassett et al. 1993), a standardized test of hand preference (Annett 1970), assessment of psychopathology (Positive and Negative Syndrome Scale; Kay et al. 1987), an assessment of disease severity (Clinical Global Impressions; Guy and Bonato 1976), and an assessment of social functioning (Global Assessment of Functioning; Endicott et al. 1976). The diagnosis was based on a consensus of two independent psychiatrists performing the Structured Clinical Interview for DSM-IV (SCID) I and II interviews (Fydrich et al. 1997; Wittchen et al. 1997). Additionally, the duration of illness (DUI; counted from the beginning of initial prodromal symptoms), the duration of psychosis (DUP; counted from the onset of diagnostic/characteristic positive symptoms), and familial risk factors (psychosis in first-degree relatives) were assessed. 
Substance use patterns and severity of substance use were assessed by the German version of the European Addiction Severity Index (EuropASI), a standardized clinical interview demonstrating high reliability and validity in patients with alcohol and drug dependence (Scheurich et al. 2000). Based on EuropASI and DSM-IV criteria of substance abuse/dependence, we divided our patient sample into two groups: Seventeen schizophrenia patients had no lifetime history of substance use disorder (SZ-NSUD; five of these had used cannabis up to five times in their lifetime), and 12 patients had accompanying substance use disorder (SZ-SUD; all cannabis use for more than 20 times in their lifetime, at least weekly consumption over a period of at least 12 weeks in the last 12 months; seven of these patients had additionally used amphetamines occasionally in their lifetime, but not in the last 3 months).

All 29 schizophrenia patients were treated with second generation antipsychotics (aripiprazole, two; olanzapine, 18; quetiapine, two; risperidone, seven; and two patients additionally treated with haloperidol). Yet, at the time of the study, no patient had been treated longer than 6 weeks continuously. To compare the cumulative and daily doses of the different antipsychotics and to explore the influence of this medication on TMS parameters, chlorpromazine (CPZ) equivalents were calculated (as suggested by reviews and studies focusing on second generation antipsychotics, e.g.,
Woods 2003). Sociodemographic and disease characteristics as well as cumulative and daily doses of antipsychotic medication (expressed in $\mathrm{CPZ}$ equivalents) are given in Table 1.

There was no concomitant treatment with benzodiazepines, mood stabilizers, beta-blocking agents, or anticholinergics in the week before TMS measurement, and no substance abuse in the 4 weeks before TMS measurement. All participants had negative quantitative urine drug screens for alcohol, tetrahydrocannabinol, amphetamines, hallucinogens, opiates, and their metabolites at the time of TMS assessment. The patients did not display any signs of withdrawal in the 4 weeks before and at the time of TMS assessment. After a complete description of the study, written informed consent was obtained from each subject. The protocol was in accordance with the Declaration of Helsinki and approved by the local ethics committee.

\section{TMS procedure}

Subjects were seated in a comfortable chair with their arms supported passively. Electromyographic (EMG) recordings from the right and left first dorsal interosseus muscle (FDI) were made with surface electrodes, using a commercial amplifier with a bandpass filter of $2 \mathrm{~Hz}$ to $10 \mathrm{kHz}$ (Keypoint portable, Medtronic Co., Denmark). Each signal

Table 1 Sociodemographic and clinical parameters of subgroups

\begin{tabular}{|c|c|c|c|c|c|c|c|c|}
\hline & \multicolumn{2}{|c|}{ SZ-NSUD $(N=17)$} & \multicolumn{2}{|c|}{ SZ-SUD $(N=12)$} & \multirow[b]{2}{*}{$\chi^{2}$} & \multicolumn{3}{|c|}{ ANOVA $^{\mathrm{a}}$} \\
\hline & $M$ & SD & $M$ & SD & & $F$ & $d f$ & $p$ value \\
\hline Age (years) & 33.6 & 7.6 & 24.4 & 6.6 & & 11.29 & 1,27 & 0.002 \\
\hline Education (years) & 11.3 & 1.7 & 9.8 & 1.5 & & 5.69 & 1,27 & 0.024 \\
\hline DUP (weeks) & 61.9 & 76.9 & 50.4 & 64.0 & & 1.35 & 1,27 & 0.26 \\
\hline DUI (weeks) & 213.9 & 184.3 & 139.7 & 99.6 & & 1.60 & 1,27 & 0.22 \\
\hline PANSS total score & 93.1 & 13.5 & 96.7 & 20.5 & & 0.33 & 1,27 & 0.57 \\
\hline PANSS positive score & 21.5 & 4.8 & 25.6 & 8.2 & & 2.83 & 1,27 & 0.10 \\
\hline PANSS negative score & 22.2 & 5.9 & 21.5 & 7.1 & & 0.08 & 1,27 & 0.78 \\
\hline PANSS gen. psychop. score & 49.4 & 7.8 & 49.6 & 11.4 & & 0.00 & 1,27 & 0.95 \\
\hline CGI & 5.9 & 0.5 & 6.1 & 0.8 & & 0.73 & 1,27 & 0.40 \\
\hline GAF & 28.8 & 8.3 & 28.3 & 13.1 & & 0.01 & 1,27 & 0.91 \\
\hline Daily dose of antipsychotics (CPZ-eq.) & 295.9 & 176.4 & 441.7 & 216.2 & & 3.99 & 1,27 & 0.06 \\
\hline Cumulative dose of antipsychotics (CPZ-eq.) & 6881.8 & 8536.4 & 8533.3 & 7112.8 & & 0.30 & 1,27 & 0.59 \\
\hline Gender (male/female $)^{\mathrm{b}}$ & $11 / 6$ & & $10 / 2$ & & 1.22 & & 1 & 0.27 \\
\hline Handedness (right/left/both) ${ }^{b}$ & $17 / 0 / 0$ & & $8 / 0 / 4$ & & 6.57 & & 1 & 0.010 \\
\hline
\end{tabular}

SZ-NSUD schizophrenia patients without cannabis abuse, $S Z$-SUD schizophrenia patients with cannabis abuse, $N$ number of patients, $M$ mean, $S D$ standard deviation, DUP duration of psychosis, DUI duration of illness (including initial prodrome), PANSS Positive and Negative Syndrome Scale, gen. psychop. general psychopathology, CGI Clinical Global Impressions, GAF Global Assessment of Functioning, CPZ-eq. chlorpromazine equivalents, $d f$ degrees of freedom, $F F$ statistics, $p$ probability

${ }^{a}$ Analysis of variance (ANOVA)

${ }^{\mathrm{b}}$ Chi-square test 
curve was manually analyzed offline. Focal TMS was applied to the hand area of the left and right motor cortex (allowing for the investigation of laterality differences) using a figure-of-eight magnetic coil and a MagPro X 100 magnetic stimulator (Medtronic Co., Denmark).

The optimal coil positions were determined for each subject individually. The optimal coil position was defined as the stimulation site that produced the largest motor evoked potential (MEP) in the resting right and left FDI muscle, respectively, at moderately suprathreshold stimulation intensities (i.e., intensities that induce MEPs of about $0.5-1.5 \mathrm{mV}, \mathrm{SI} 1 \mathrm{mV})$. Sites were marked to ensure constant coil position throughout the experiment. The coil was held tangentially to the head, with the handle pointing backwards and away from the midline at a $45^{\circ}$ lateral angle. This ensured that the induced current pointed forwards and perpendicular to the central sulcus, which is optimal for producing transsynaptic activation of corticospinal neurons. The resting motor threshold (RMT), expressed as a percentage of maximum stimulator output, was defined as the lowest intensity that produced an MEP of $>50 \mu \mathrm{V}$ in at least five out of ten trials in the relaxed FDI (Ziemann et al. 1996a).

SICI and ICF were assessed using standard paired-pulse procedures (e.g., Eichhammer et al. 2004; Maeda and Pascual-Leone 2003). The intensity of the first (conditioning) stimulus was always set to $80 \%$ of the RMT. The second (test) stimulus was delivered at an intensity that produced MEPs averaging $0.5-1.5 \mathrm{mV}$ in the resting FDI $(\mathrm{SI} 1 \mathrm{mV})$. Based on previous research showing that short inter-stimulus intervals (ISIs) of $2-5 \mathrm{~ms}$ lead to inhibition and longer ISIs $(7-20 \mathrm{~ms})$ lead to facilitation of test stimulus MEPs (Kujirai et al. 1993), we used an ISI of $3 \mathrm{~ms}$ in the inhibitory (SICI) and an ISI of $15 \mathrm{~ms}$ in the facilitatory (ICF) paired-pulse TMS paradigm. We performed a minimum of ten paired-pulse trials at each ISI and at least tne single-pulse trials with an unconditioned test stimulus. The effect of the conditioning stimulus on MEP amplitude of the test stimulus was determined as the ratio of the average amplitude of the conditioned test MEP (cMEP) to the average amplitude of the unconditioned test MEP (uMEP).

Measurement of the CSP duration was obtained with moderately tonically active FDI $(25-30 \%$ of maximal contraction) by stimulating the contralateral motor cortex with intensities of $120 \%, 140 \%, 160 \%$, and $180 \%$ of RMT. For each intensity, 16 trials were performed and the mean CSP duration calculated. The CSP duration was defined as the time from MEP onset to the return of voluntary EMG activity (absolute CSP; Daskalakis et al. 2003).

The measurements were performed by an experienced investigator, controlled by another experienced investigator, and corrected for outliers and extreme values. Trials were ordered quasi-randomly for each subject, and the order was counterbalanced across groups. The investigator analyzing the data was blinded to group status.

\section{Statistics}

For statistical analyses, SPSS for Windows 15.0 was used. All tests were two-tailed. Level of significance was set at $\alpha=0.05$. Dependent variables were RMT, SICI (ISI $3 \mathrm{~ms}$ ), ICF (ISI $15 \mathrm{~ms}$ ), and contralateral CSP (intensities $120 \%, 140 \%$, $160 \%$, and $180 \%$ of RMT) of both hemispheres. The independent variable was SUD diagnosis (SZ-SUD and SZ-NSUD).

One-way analysis of variance (ANOVA) and chi-squared tests of independence were used to analyze differences between the two diagnostic groups on demographic variables age, education level, gender, and dexterity, as well as clinical measures.

The primary analyses focused on SUD-group differences in TMS measures. After applying Kolmogorov-Smirnov tests and confirming normal distribution for RMT and CSP variables, analysis of covariance (ANCOVA; with intervening variables gender, hand preference, age, education level, and CPZ equivalents) was used to test for significant differences in CSP duration and RMT intensities between the diagnostic groups. As the normality assumption was violated for SICI and ICF, non-parametric tests (MannWhitney $U$ tests) were performed for these variables.

Laterality effects were assessed as follows. For RMT, a repeated measures MANOVA (within-subject factor hemisphere, between-subject factor SUD diagnosis) was performed to test the hypothesis that in right-handed subjects RMT is lower in the left versus right motor cortex. The Wilcoxon rank test was used to detect differences between SICI/ICF measures after left and right hemispheric stimulation.

Finally, Spearman rank correlations between SICI/ICF variables and CPZ equivalents, total Positive and Negative Syndrome Scale (PANSS), Global Assessment of Functioning (GAF), Clinical Global Impression (CGI), DUP, and DUI scores were computed across groups and for each group separately.

\section{Results}

Sociodemographic and clinical characteristics

SZ-SUD subjects were significantly younger than SZNSUD subjects (mean age, $24.4 \pm 6.6$ years versus $33.6 \pm$ 7.6 years, $p=0.002)$. They also had a significantly lower education level $(9.8 \pm 1.5$ versus $11.3 \pm 1.7$ years, $p=0.024)$. The proportion of male patients was significantly higher in the SZ-SUD group $(p=0.023)$. While all SZ-NSUD 
subjects were right handed, four patients of the SZ-SUD group were ambidexters.

The patients suffered from moderate to severe positive and negative symptoms according to the PANSS, accompanied by a severe degree of illness (CGI) and severe impairment of social functioning (GAF). There were no group differences in psychopathology (PANSS), disease severity (CGI), global functioning (GAF), DUI (prodrome), or DUP (for details see Table 1).

No subject fulfilled the criteria for borderline or antisocial personality disorder (according to the SCID II interview, DSM-IV axis II, personality disorders), which include abnormally high impulsivity, which in turn is potentially linked to reduced inhibition processes. The dosage of daily and cumulative antipsychotic medication before TMS assessment (expressed in CPZ equivalents) did not differ significantly between the two groups, despite a trend toward higher daily dosage in the SZ-SUD group (441.67 versus 295.88; $p=$ 0.06). Across groups, more severely ill patients received higher medication dosages: the cumulative doses and total PANSS scores correlated positively at a trend level $(\mathrm{rho}=$ $0.34, p=0.068$ ), and the correlation of cumulative dosage and CGI was significant (rho $=0.65, p<0.0005)$.

\section{Resting motor threshold}

RMT in both hemispheres was lower in SZ-SUD (left motor cortex, 44.91\%; right motor cortex, 47.0\%) compared to SZ-NSUD subjects (left motor cortex, 48.18\%; right motor cortex, $49.06 \%$ ), but this difference was not significant (see Table 2). The repeated measures MANOVA (within-subject factor hemisphere, between-subject factor SUD diagnosis) revealed no significant hemisphere effects and in particular no interaction; hence, there was no evidence for RMT asymmetry between groups.

Short-interval intracortical inhibition and intracortical facilitation

SZ-SUD patients showed a reduced SICI (ISI $3 \mathrm{~ms} ; 65.3 \%$ lower inhibition stimulating right motor cortex; $36.3 \%$ lower inhibition after stimulation of the left motor cortex) and increased ICF (ISI $15 \mathrm{~ms} ; 81.3 \%$ increased facilitation stimulating right motor cortex; $50.8 \%$ increased facilitation after stimulation of the left motor cortex) as compared to SZ-NSUD patients. The reduction of SICI $(Z=-2.2, d f=1$, $p=0.026$; Mann-Whitney $U$ test) and the increase of ICF $(Z=-2.0, d f=1, p=0.047$; Mann-Whitney $U$ test) were both significant following right motor cortex stimulation (see Table 2, Figs. 1 and 2). Reduction of SICI and increase of ICF in SZ-SUD compared to SZ-NSUD patients were not significant when stimulating the left primary motor cortex (for details see Table 2).
Since there were significant differences in age, education, and hand preference, and also a trend level difference in daily dosage of antipsychotic medication, we performed an additional ANCOVA (factor SUD diagnosis; intervening variables gender, age, education level, and daily dosage of antipsychotic medication). This was to rule out the possibility that the significant results of the non-parametric tests were due to these subgroup differences. With this approach, again, there was a significant reduction of SICI $[F(1,19)=19.1, p<0.001]$ and a significant increase of ICF $[F(1,19)=12.2, p=0.003]$ in SZ-SUD compared to SZ-NSUD subjects.

Neither in the total sample nor in the subgroups were there any differences in SICI/ICF measures comparing left and right hemispheric stimulation (Wilcoxon rank test).

\section{Cortical silent period}

Increased stimulation intensity $(120 \%, 140 \%, 160 \%$, and $180 \%$ RMT) resulted in longer CSP duration in both groups, as expected. No significant group differences were found in CSP after either right or left motor cortex stimulation (ANCOVA; factor SUD diagnosis; intervening variables gender, age, education level, hand preference, and daily dosage of antipsychotic medication; for details see Table 2).

Looking at overall hemispheric differences, CSP duration tended to be longer after right motor cortex stimulation at $180 \%$ RMT intensity (left FDI; $p=0.083$, MANOVA, within-subject factor hemisphere). Looking at hemispheric difference effects between groups (effectively the hemisphere $\times$ group interaction), the hemispheric CSP difference (CSP left FDI-CSP right FDI) at 120\% RMT stimulation intensity was positive (left $>$ right FDI) for SZ-NSUD and negative (right $>$ left FDI) for SZ-SUD patients. The corresponding hemisphere by group ANOVA returned a significant interaction effect $[F(1,26)=4.10, p=0.054]$. That is, SZ-SUD patients displayed a slightly longer CSP after left motor cortex stimulation (right FDI) and a shorter CSP after right motor cortex stimulation (left FDI) than SZNSUD patients. We also calculated a CSP duration asymmetry index at $120 \%$ RMT [AI $=2$ (right - left) (right + left)] and found this to be negative in SZ-NSUD patients $(-0.0568)$ and positive in SZ-SUD patients $(+0.1849)$. This is a significant difference $[F(1,26)=4.28, p=0.049$; ANOVA $]$, although it would not survive adjustment for multiple testing (Bonferroni correction).

Influence of clinical variables and antipsychotic medication on TMS parameters

To detect any influence of clinical variables or medication on SICI/ICF, we calculated non-parametric correlations (Spearman rank correlations) between these variables and TMS 
Table 2 Comparison of TMS parameters between subgroups

\begin{tabular}{|c|c|c|c|c|c|c|c|c|c|c|}
\hline & \multicolumn{2}{|c|}{$\begin{array}{l}\text { SZ-NSUD } \\
(N=17)\end{array}$} & \multicolumn{2}{|c|}{$\begin{array}{l}\text { SZ-SUD } \\
(N=12)\end{array}$} & \multicolumn{3}{|c|}{ ANCOVA $^{\mathrm{c}}$} & \multicolumn{3}{|c|}{$\begin{array}{l}\text { Mann-Whitney } \\
U \text {-Test }\end{array}$} \\
\hline & $M$ & SD & $M$ & SD & $d f$ & $F$ & $p$ value & $d f$ & $Z$ & $p$ value \\
\hline RMT left motor cortex $(\%)^{\mathrm{a}}$ & 48.18 & 7.30 & 44.91 & 6.35 & 1,19 & 0.26 & 0.62 & & & \\
\hline RMT right motor cortex $(\%)^{\mathrm{a}}$ & 49.06 & 8.41 & 47.00 & 7.66 & 1,19 & 0.35 & 0.56 & & & \\
\hline \multicolumn{11}{|l|}{ ppTMS } \\
\hline $\begin{array}{l}\text { SICI (ISI } 3 \mathrm{~ms} \text { ) left motor cortex } \\
\text { (ratio cMEP/uMEP) }^{\mathrm{b}}\end{array}$ & 0.36 & 0.33 & 0.49 & 0.32 & & & & 1 & -1.5 & 0.14 \\
\hline $\begin{array}{l}\text { SICI (ISI } 3 \mathrm{~ms} \text { ) right motor cortex } \\
\text { (ratio cMEP/uMEP) }^{\mathrm{b}}\end{array}$ & 0.41 & 0.40 & 0.67 & 0.68 & & & & 1 & -2.2 & 0.026 \\
\hline $\begin{array}{l}\text { ICF (ISI } 15 \mathrm{~ms} \text { ) left motor cortex } \\
\text { (ratio cMEP/uMEP) }^{\mathrm{b}}\end{array}$ & 1.41 & 1.07 & 2.13 & 1.63 & & & & 1 & -1.6 & 0.13 \\
\hline $\begin{array}{l}\text { ICF (ISI } 15 \mathrm{~ms} \text { ) right motor cortex } \\
\text { (ratio cMEP/uMEP) }^{\mathrm{b}}\end{array}$ & 1.48 & 1.18 & 2.67 & 1.99 & & & & 1 & -2.0 & 0.047 \\
\hline \multicolumn{11}{|l|}{ Cortical silent period } \\
\hline CSP right FDI 120\% RMT (ms) & 159.81 & 48.81 & 169.91 & 23.63 & 1,21 & 0.2 & 0.65 & & & \\
\hline CSP right FDI $140 \%$ RMT (ms) & 207.83 & 47.04 & 208.04 & 32.98 & 1,19 & 0.3 & 0.60 & & & \\
\hline CSP right FDI $160 \%$ RMT (ms) & 235.27 & 40.85 & 237.75 & 31.24 & 1,21 & 0.9 & 0.35 & & & \\
\hline CSP right FDI 180\% RMT (ms) & 239.69 & 30.38 & 257.31 & 39.00 & 1,16 & 1.6 & 0.22 & & & \\
\hline CSP left FDI $120 \%$ RMT (ms) & 171.84 & 59.68 & 145.05 & 43.77 & 1,22 & 0.0 & 0.99 & & & \\
\hline CSP left FDI 140\% RMT (ms) & 211.64 & 63.75 & 213.50 & 46.18 & 1,19 & 0.7 & 0.42 & & & \\
\hline CSP left FDI 160\% RMT (ms) & 240.61 & 51.10 & 246.27 & 41.45 & 1,19 & 1.2 & 0.29 & & & \\
\hline CSP left FDI 180\% RMT (ms) & 255.54 & 61.80 & 268.19 & 21.92 & 1,16 & 0.1 & 0.93 & & & \\
\hline
\end{tabular}

SZ-NSUD schizophrenia patients without cannabis abuse, $S Z-S U D$ schizophrenia patients with cannabis abuse, $M$ mean, $S D$ standard deviation, $N$ number of patients, $d f$ degrees of freedom, $F F$ statistics, $p$ probability, $Z Z$ value, $c M E P$ conditioned motor evoked potential, $u M E P$ unconditioned motor evoked potential, RMT resting motor threshold, ANCOVA analysis of covariance, CSP contralateral cortical silent period, FDI first dorsal interosseus muscle, $m s$ milliseconds

${ }^{\text {a }}$ Percentage of maximum stimulator output

${ }^{\mathrm{b}}$ As ppTMS variables were not normally distributed, non-parametric testing (Mann-Whitney $U$ test) was used

${ }^{\mathrm{c}}$ Age, education, and chlorpromazine-equivalents were included as covariates

parameters. No significant correlations between psychopathology (PANSS), disease severity (CGI), social functioning (GAF), DUP, or DUI and TMS parameters (SICI, ICF, and CSP) were observed. In addition, the duration of cannabis abuse (weeks of consumption) and the age of cannabis abuse onset were not significantly correlated with either SICI or ICF.

As outlined before, SZ-SUD patients tended to receive a higher daily dose (CPZ equivalents) of antipsychotic medication than SZ-NSUD patients. While there was no significant correlation between SICI and cumulative or daily dose of antipsychotics, in the SZ-SUD group, there was trend-level association between the cumulative antipsychotic dose and ICF following right motor cortex stimulation ( $\mathrm{rho}=0.517, p=$ 0.05 ) but not left motor cortex stimulation.

\section{Discussion}

The present study used TMS to investigate motor cortical excitability in schizophrenia patients with and without comorbid SUD (cannabis). The TMS technique allows noninvasive testing of inhibitory and excitatory intracortical networks in the human brain. In contrast to most animal research, TMS can describe alterations at the system level of the human cerebral cortex; this level typically has a closer relation to the clinical context (Lang et al. 2008).

In a previous study, we were able to identify an SICI reduction in a sample of first-episode schizophrenia patients relative to healthy control subjects. This suggested that a GABAergic deficit may be involved in schizophrenic pathophysiology, which would support the functional dysconnectivity hypothesis (Wobrock et al. 2008). Functional disconnectivity in schizophrenia-as indexed by TMS measures - has also been found between posterior parietal cortex and ipsilateral motor cortex (Koch et al. 2008). To our knowledge, our study is the first to use TMS to explore the effects of accompanying cannabis abuse on cortical excitability in schizophrenia.

The main result of our study is that schizophrenia patients with a history of cannabis abuse (SZ-SUD) showed 


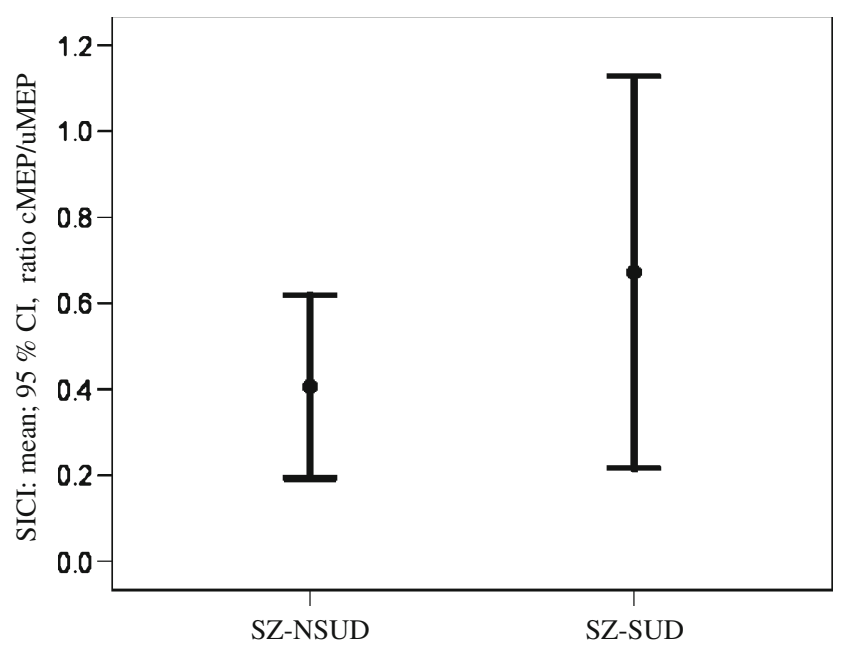

Fig. 1 Short interval cortical inhibition (SICI) in the schizophrenia subgroups. First-episode schizophrenia patients with comorbid cannabis abuse (SZ-SUD) showed significantly reduced cortical inhibition (SICI; inhibitory ISI of $3 \mathrm{~ms}$; higher ratio, conditioned MEP/ unconditioned MEP; mean, 95\% CI) compared to patients without previous cannabis abuse (SZ-NSUD) in right motor cortex $(Z=-2.2$, $p=0.026$; Mann-Whitney $U$ test). $C I$ confidence interval, $c M E P$ conditioned motor evoked potential, $u M E P$ unconditioned motor evoked potential, ISI interstimulus interval, SZ-NSUD patients with first-episode schizophrenia without previous cannabis abuse, $S Z-S U D$ patients with first-episode schizophrenia and comorbid cannabis abuse

a reduced $\mathrm{GABA}_{\mathrm{A}}$-mediated SICI and an enhanced ICF compared to patients with no cannabis abuse history (SZNSUD). The $\mathrm{GABA}_{\mathrm{B}}$-mediated CSP and parameters of corticospinal excitability such as RMT and SI $1 \mathrm{mV}$ did not differ between the groups. We found no significant hemispheric differences in TMS measures (after Bonferroni correction).

In our study, SZ-SUD patients were significantly younger and less educated than SZ-NSUD patients. This is in accordance with other cross-sectional studies (CantorGraae et al. 2001; Mueser et al. 1990; Wobrock and Soyka 2008); therefore, our patient samples seem to be representative for this special patient population. Importantly, psychopathology and disease severity did not differ between the two schizophrenia subgroups.

A number of TMS studies have now found reduced intracortical inhibition and enhanced ICF in schizophrenia patients (Daskalakis et al. 2002; Eichhammer et al. 2004; Fitzgerald et al. 2002a,b,c; Pascual-Leone et al. 2002; Wobrock et al. 2008). SICI seems to be induced by GABAergic interneurons via $\alpha 2-$ or $\alpha 3-\mathrm{GABA}_{\mathrm{A}}$ receptors (Di Lazzaro et al. 2007; Siebner et al. 1998), whereas ICF relates to the excitability of excitatory neuronal circuits in motor cortex; these circuits are at least partially dissociable from the SICI network (Ziemann 2004). In schizophrenia, GABAergic dysfunction, decreased levels of the mRNA expression of the GABA-synthesizing enzyme glutamic acid decarboxylase (GAD67), and a reduction of GABAergic interneurons are all observed (Benes 1998; Knable et al. 2002; Lewis et al. 2005). Furthermore, expression levels of seven GABA-related transcripts have been found to be reduced in subjects with schizophrenia in four cortical regions (including the motor cortex); these changes may cause cortical dysfunction in schizophrenia patients (Hashimoto et al. 2008).

These neuropathological and neurobiological findings are in line with the reduced SICI observed in schizophrenia. Our results most likely reflect an amplified GABAergic deficit and a distinct functional intracortical disconnectivity in cannabis-abusing schizophrenia patients. Our data demonstrate, for the first time, the influence of previous cannabis abuse on cortical GABAergic function in schizophrenia patients.

The influence of cannabis consumption on TMS measures in subjects not suffering from schizophrenia was investigated in a recently published study by Fitzgerald et al. (2009). These authors examined 42 chronic cannabis users (both heavy and light users) and observed reduced SICI relative to healthy controls, without any differences in CSP. This finding corroborates our view that chronic cannabis use may cause a $\mathrm{GABA}_{\mathrm{A}}$-mediated reduction of intracortical inhibition without any influence on $\mathrm{GABA}_{\mathrm{B}}$ activity.

In summary, there is convincing evidence for a link between cannabis, schizophrenia, and the GABAergic

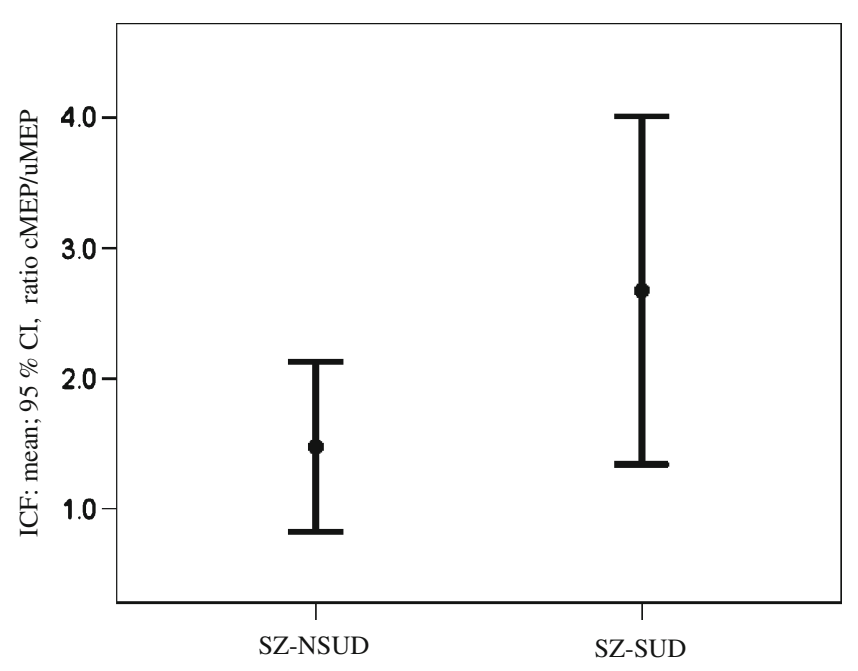

Fig. 2 Intracortical facilitation (ICF) in the schizophrenia subgroups. First-episode schizophrenia patients with comorbid cannabis abuse (SZ-SUD) showed significantly enhanced facilitation (facilitatory ISI of $15 \mathrm{~ms}$; higher ratio, conditioned MEP/unconditioned MEP; mean, $95 \% \mathrm{CI}$ ) compared to patients without previous cannabis abuse (SZNSUD) in right motor cortex $(Z=-2.0, p=0.047$; Mann-Whitney $U$ test). $C I$ confidence interval, $c M E P$ conditioned motor evoked potential, $u M E P$ unconditioned motor evoked potential, ISI interstimulus interval, $S Z-N S U D$ patients with first-episode schizophrenia without previous cannabis abuse, SZ-SUD patients with first-episode schizophrenia and comorbid cannabis abuse 
system. In this vein, our results are also in line with studies reporting a reduction of event-related potentials - such as the auditory evoked P50 or P300 - in both schizophrenia, including its prodrominal states, and after cannabis use (Adler et al. 1982; Coburn et al. 1998; Frommann et al. 2008; Ozgürdal et al. 2008; Roser et al. 2008), probably also reflecting reduced inhibition in a cortico-subcortical loop.

The active compound of herbal cannabis, delta-9tetrahydrocannabinol (THC), exerts all of its known central effects through CB1Rs. Cannabinoids, via CB1Rs, influence the release of glutamate, dopamine, acetylcholine, and GABA primarily presynaptically. In contrast, the cellular mechanisms involved in the inhibition of neurotransmitter release by cannabinoids are still not fully understood (Iversen 2003). Several mechanisms are suspected to be engaged in these inhibitory effects of CB1R activation, including activation of $\mathrm{N}$-type $\mathrm{Ca}^{+}{ }^{+}$currents (Caulfield and Brown 1992), a reduction of the frequency of $\mathrm{Ca}_{2}^{+}-$ independent excitatory or inhibitory synaptic currents (Schlicker and Kathmann 2001), and decreased phosphorylation of A-type $\mathrm{K}^{+}$channels by the cAMP-dependent enzyme protein kinase A (Deadwyler et al. 1995). In the rodent hippocampus, a specific class of GABAergic interneurons was detected to express CB1Rs, and moreover, endogenous and exogenous cannabinoids were shown to inhibit GABA release in CA1 pyramidal cells (Hoffman and Lupica 2000; Katona et al. 1999, 2000) and pyramidal cells of the ventral tegmental area (Pistis et al. 2001).

In our study, patients with a double diagnosis showed an enhanced ICF. Likewise, atropine, a cholinergic antagonist, has been found to enhance ICF (Liepert et al. 2001), while NMDA antagonists and chronic nicotine exposure have been associated with an ICF reduction (Lang et al. 2008; Schwenkreis et al. 1999; Ziemann et al. 1998). The enhanced ICF in schizophrenia patients with comorbid cannabis abuse might hence best be explained by an increased glutamatergic input and reduced $\mathrm{GABA}_{\mathrm{A}}$ ergic neurotransmission, while a disturbance in cholinergic intracortical neuronal circuits may also play a role.

Previous studies investigating the action of addictive drugs (other than cannabis) on cortical excitability have revealed partially conflicting results. Chronic nicotine abuse, acute alcohol exposure, and alcohol dependence have been associated with enhanced inhibition and reduced facilitation in specific neuronal circuits in motor cortex (Conte et al. 2007; Lang et al. 2008; Ziemann et al. 1996b). Cocaine addicts showed decreased excitability of the motor cortex (Boutros et al. 2005), whereas ecstasy users demonstrated increased excitability of the visual cortex (Oliveri and Calvo 2003). Apart from the specific type of drug under investigation, there are two likely reasons why the observed effects of drug modulation contrast with the effects reported in the present study: First, effects may critically depend on specific neurodevelopmental brain abnormalities in schizophrenia patients (Falkai et al. 2008). Secondly, most of the TMS studies investigating addictive drug effects on cortical excitability have looked at acute drug effects or have tested subjects that were under the influence of the drug at the time of TMS measurement. In our sample, we assessed patients in a drug-free interval, to investigate the pathophysiology of this double diagnosis condition without the influence of acute drug effects or long-lasting intoxication effects.

However, our study has several limitations. Although we collected data about the pattern of substance abuse, we did not differentiate between different types of cannabis or the average amount of cannabis per use. The content of psychoactive THC varies widely across different types of cannabis (Potter et al. 2008). This complicates the exploration of dose-response relationships, although in our sample, we found no correlation between TMS parameters and the amount of cannabis consumption (lifetime days of use).

Secondly, antipsychotic medication could be a considerable confounding factor in TMS paradigms (Davey et al. 1997). For instance, one study found a difference in RMT between patients treated with risperidone (increased RMT) and olanzapine (decreased RMT; Fitzgerald et al. 2002c). For paired-pulse measurements, medication influencing GABA, glutamate, serotonin, or dopamine transmission could be potentially relevant because the interneuronalpyramidal circuits are mediated via these pathways. However, according to the literature, one would expect that antipsychotics should compensate for the reduced inhibition or increased facilitation and should not produce it, as there are no studies available showing increased cortical inhibition in medicated (or unmedicated) schizophrenia and only one study demonstrating reduced cortical inhibition exclusively in medicated patients (Pascual-Leone et al. 2002). In our study, we could not rule out an influence of antipsychotic dosing on ICF completely, as we detected a positive correlation between the extent of facilitation (MEP ratio) and the cumulative antipsychotic dosage in the SZSUD patient group.

Furthermore, although we consider our patient sample to be quite representative, groups differed in central demographic parameters (gender, age, and education). This may have affected our main results, although we consider this unlikely, as we adjusted our analyses for confounding variables. Another limitation is that we did not determine the concentration of endogenous cannabinoids (e.g., anandamide) in the CSF, as it has been done in previous studies with cannabis abusing schizophrenia patients (Leweke et al. 2007). It would have been interesting to see if dysregulations of the endogenous cannabinoid system contributed to the observed GABAergic dysfunction as assessed by TMS. 
Finally, we did not administer THC in a controlled fashion and therefore cannot conclude directly that the cortical excitability differences between the groups under investigation were caused by cannabis consumption. Alternatively, the excitability differences could represent a kind of brain dysfunction that precedes clinical diagnosis of schizophrenia and increases the risk for SUDs. We do not consider this alternative interpretation likely.

The strength of our study is that we were able to investigate a sample of first-episode patients that was both well-characterized and representative for this patient population in a clinical setting. Due to disease severity and the clinical necessity for medication, it was of course not possible to test these patients in a neuroleptic-naive setting.

Summing up, this study affords novel insights into the cortical physiology of schizophrenia patients with comorbid cannabis use disorder. The etiological relationship between the two diagnoses is not yet fully understood, but this study supports the theory that cannabis abuse acts as a distinct vulnerability factor for schizophrenia through an increase in GABAergic dysfunction. Alternatively, our results support the hypothesis of an increased sensitivity to the effects of drugs in this special population.

Acknowledgments The authors declare that, except income received from primary employers, no financial support or compensation has been received from any individual or corporate entity for research or professional service related to the presented work/manuscript, and there are no personal finance holdings that could be perceived as constituting a potential conflict of interest.

Open Access This article is distributed under the terms of the Creative Commons Attribution Noncommercial License which permits any noncommercial use, distribution, and reproduction in any medium, provided the original author(s) and source are credited.

\section{References}

Abi-Dargham A, Rodenhiser J, Printz D, Zea-Ponce Y, Gil R, Kegeles LS, Weiss R, Cooper TB, Mann JJ, Van Heertum RL, Gorman JM, Laruelle M (2000) Increased baseline occupancy of D2 receptors by dopamine in schizophrenia. Proc Natl Acad Sci USA 97:8104-8109

Adler LE, Pachtman E, Frank RD, Pecevich M, Waldo MC, Freedman R (1982) Neurophysiological evidence for a defect in neuronal mechanisms involved in sensory gating in schizophrenia. Biol Psychiatry 17:639-654

Annett M (1970) A classification of hand preference by association analysis. Br J Psychol 61:303-321

Bassett AS, Collins EJ, Nuttall SE, Honer WG (1993) Positive and negative symptoms in families with schizophrenia. Schizophr Res 11:9-19

Benes FM (1998) Model generation and testing to probe neural circuitry in the cingulate cortex of postmortem schizophrenic brain. Schizophr Bull 24:219-230

Boutros NN, Lisanby SH, McClain-Furmanski D, Oliwa G, Gooding D, Kosten TR (2005) Cortical excitability in cocaine-dependent patients: a replication and extension of TMS findings. J Psychiatr Res 39(3):295-302

Cantor-Graae E, Nordstrom LG, McNeil TF (2001) Substance abuse in schizophrenia: a review of the literature and a study of correlates in Sweden. Schizophr Res 48:69-82

Caulfield MP, Brown DA (1992) Cannabinoid receptor agonists inhibit Ca current in NG108-15 neuroblastoma cells via a pertussis toxinsensitive mechanism. Br J Pharmacol 106(2):231-232

Coburn KL, Shillcutt SD, Tucker KA, Estes KM, Brin FB, Merai P, Moore NC (1998) P300 delay and attenuation in schizophrenia: reversal by neuroleptic medication. Biol Psychiatry 44(6):466474

Conte A, Attilia ML, Gilio F, Iacovelli E, Frasca V, Bettolo CM, Gabriele M, Giacomelli E, Prencipe M, Berardelli A, Ceccanti M, Inghilleri M (2007) Acute and chronic effects of ethanol on cortical excitability. Clin Neurophysiol 119(3):667-674

Daskalakis ZJ, Christensen BK, Chen R, Fitzgerald PB, Zipursky RB, Kapur S (2002) Evidence for impaired cortical inhibition in schizophrenia using transcranial magnetic stimulation. Arch Gen Psychiatry 59(4):347-354

Daskalakis ZJ, Christensen BK, Chen R, Fitzgerald PB, Zipursky RB, Kapur S (2003) Effect of antipsychotics on cortical inhibition using transcranial magnetic stimulation. Psychopharmacology (Berl) 170(3):255-262

Davey NJ, Puri BK, Lewis HS, Lewis SW, Ellaway PH (1997) Effects of antipsychotic medication on electromyographic responses to transcranial magnetic stimulation of the motor cortex in schizophrenia. J Neurol Neurosurg Psychiatry 63(4):468-473

Deadwyler SA, Hampson RE, Mu J, Whyte A, Childers S (1995) Cannabinoids modulate voltage sensitive potassium A-current in hippocampal neurons via a cAMP-dependent process. J Pharmacol Exp Ther 273(2):734-743

Dean B, Sundram S, Bradbury R, Scarr E, Copolov D (2001) Studies on $[3 \mathrm{H}] \mathrm{CP}-55940$ binding in the human central nervous system: regional specific changes in density of cannabinoid-1 receptors associated with schizophrenia and cannabis use. Neuroscience 103(1):9-15

Di Lazzaro V, Pilato F, Dileone M, Profice P, Ranieri F, Ricci V, Bria P, Tonali PA, Ziemann U (2007) Segregating two inhibitory circuits in human motor cortex at the level of $\mathrm{GABA}_{\mathrm{A}}$ receptor subtypes: a TMS study. Clin Neurophysiol 118(10):2207-2214

Eggan SM, Hashimoto T, Lewis DA (2008) Reduced cortical cannabinoid 1 receptor messenger RNA and protein expression in schizophrenia. Arch Gen Psychiatry 65(7):772-784

Eichhammer P, Wiegand R, Kharraz A, Langguth B, Binder H, Hajak G (2004) Cortical excitability in neuroleptic-naive first-episode schizophrenic patients. Schizophr Res 67(2-3):253-259

Endicott J, Spitzer R, Fleiss JL, Cohen J (1976) The global assessment scale: a procedure for measuring overall severity of psychiatric disturbance. Arch Gen Psychiatry 33:766-771

Falkai P, Wobrock T, Schneider-Axmann T, Gruber O (2008) Schizophrenia as a brain disorder and its development. Fortschr Neurol Psychiatr 76(Suppl 1):63-67

Fitzgerald PB, Brown TL, Daskalakis ZJ, deCastella A, Kulkarni J (2002a) A study of transcallosal inhibition in schizophrenia using transcranial magnetic stimulation. Schizophr Res 56(3):199-209

Fitzgerald PB, Brown TL, Daskalakis ZJ, Kulkarni J (2002b) A transcranial magnetic stimulation study of inhibitory deficits in the motor cortex in patients with schizophrenia. Psychiatry Res 114(1):11-22

Fitzgerald PB, Brown TL, Daskalakis ZJ, Kulkarni J (2002c) A transcranial magnetic stimulation study of the effects of olanzapine and risperidone on motor cortical excitability in patients with schizophrenia. Psychopharmacology (Berl) 162(1):74-81

Fitzgerald PB, Williams S, Daskalakis ZJ (2009) A transcranial magnetic stimulation study of the effects of cannabis use on 
motor cortical inhibition and excitability. Neuropsychopharmacology 34(11):2368-2375

Frommann I, Brinkmeyer J, Ruhrmann S, Hack E, Brockhaus-Dumke A, Bechdolf A, Wölwer W, Klosterkötter J, Maier W, Wagner M (2008) Auditory P300 in individuals clinically at risk for psychosis. Int J Psychophysiol 70(3):192-205

Fydrich T, Renneberg B, Schmitz B, Wittchen HU (1997) Strukturiertes Klinisches Interview für DSM-IV. Achse II: Persönlichkeitsstörungen. A German, advanced adaptation of the original SCID version by RL Spitzer, JB Williams, M Gibbon and MB First. Hogrefe-Verlag, Göttingen

Giuffrida A, Leweke FM, Gerth CW, Schreiber D, Koethe D, Faulhaber J, Klosterkötter J, Piomelli D (2004) Cerebrospinal anandamide levels are elevated in acute schizophrenia and are inversely correlated with psychotic symptoms. Neuropsychopharmacology 29(11):2108-2114

Guy W, Bonato RR (1976) CGI: Clinical global impressions. In: Manual for the ECDEU assessment battery.2. Rev edn. National Institute of Mental Health, Chevy Chase, pp 12/1-12/6

Hashimoto T, Bazmi HH, Mirnics K, Wu Q, Sampson AR, Lewis DA (2008) Conserved regional patterns of GABA-related transcript expression in the neocortex of subjects with schizophrenia. Am J Psychiatry 165(4):479-489

Hoffman AF, Lupica CR (2000) Mechanisms of cannabinoid inhibition of GABA(A) synaptic transmission in the hippocampus. J Neurosci 20(7):2470-2479

Hoffman AF, Lupica CR (2001) Direct actions of cannabinoids on synaptic transmission in the nucleus accumbens: a comparison with opioids. J Neurophysiol 85:72-83

Iversen L (2003) Cannabis and the brain. Brain 126(Pt 6):1252-1270

Katona I, Sperlágh B, Sík A, Käfalvi A, Vizi ES, Mackie K, Freund TF (1999) Presynaptically located CB1 cannabinoid receptors regulate GABA release from axon terminals of specific hippocampal interneurons. J Neurosci 19(11):4544-4558

Katona I, Sperlágh B, Maglóczky Z, Sántha E, Köfalvi A, Czirják S, Mackie K, Vizi ES, Freund TF (2000) GABAergic interneurons are the targets of cannabinoid actions in the human hippocampus. Neuroscience 100(4):797-804

Kay SR, Fiszbein A, Opler LA (1987) The Positive and Negative Syndrome Scale (PANSS) for schizophrenia. Schizophr Bull 13:261-276

Knable MB, Barci BM, Bartko JJ, Webster MJ, Torrey EF (2002) Molecular abnormalities in the major psychiatric illnesses: classification and regression tree (CRT) analysis of post-mortem prefrontal markers. Mol Psychiatry 7:392-404

Koch G, Ribolsi M, Mori F, Sacchetti L, Codecà C, Rubino IA, Siracusano A, Bernardi G, Centonze D (2008) Connectivity between posterior parietal cortex and ipsilateral motor cortex is altered in schizophrenia. Biol Psychiatry 64(9):815-819

Kujirai T, Caramis MD, Rothwell JC, Day BL, Thompson PD, Ferbert A, Wroe S, Asselman P, Arsden CD (1993) Corticospinal inhibition in human motor cortex. J Physiol 471:501-519

Lang N, Hasan A, Sueske E, Paulus W, Nitsche MA (2008) Cortical hypoexcitability in chronic smokers? A transcranial magnetic stimulation study. Neuropsychopharmacology 33(10):2517-2523

Leweke FM, Giuffrida A, Koethe D, Schreiber D, Nolden BM, Kranaster L, Neatby MA, Schneider M, Gerth CW, Hellmich M, Klosterkötter J, Piomelli D (2007) Anandamide levels in cerebrospinal fluid of first-episode schizophrenic patients: impact of cannabis use. Schizophr Res 94(1-3):29-36

Lewis DA, Hashimoto T, Volk DW (2005) Cortical inhibitory neurons and schizophrenia. Nat Rev Neurosci 6:312-324

Liepert J, Schardt S, Weiler C (2001) Orally administred atropine enhances motor cortex excitability: a transcranial magnetic stimulation study in human subjects. Neurosci Lett 300:149-152
Maeda F, Pascual-Leone A (2003) Transcranial magnetic stimulation: studying motor neurophysiology of psychiatric disorders. Psychopharmacology 168:359-376

Mueser KT, Yarnold PR, Levinson DR, Singh H, Bellack AS, Kee K, Morrison RL, Yadalam KG (1990) Prevalence of substance abuse in schizophrenia: demographic and clinical correlates. Schizophr Bull 16:31-56

Oliveri M, Calvo G (2003) Increased visual cortical excitability in ecstasy users: a transcranial magnetic stimulation study. J Neurol Neurosurg Psychiatry 74(8):1136-1138

Ozgürdal S, Gudlowski Y, Witthaus H, Kawohl W, Uhl I, Hauser M, Gorynia I, Gallinat J, Heinze M, Heinz A, Juckel G (2008) Reduction of auditory event-related P300 amplitude in subjects with at-risk mental state for schizophrenia. Schizophr Res 105(13):272-278

Pascual-Leone A, Manoach DS, Birnbaum R, Goff DC (2002) Motor cortical excitability in schizophrenia. Biol Psychiatry 52(1):24 31

Pistis M, Porcu G, Melis M, Diana M, Gessa GL (2001) Effects of cannabinoids on prefrontal neuronal responses to ventral tegmental area stimulation. Eur J Neurosc 14:96-102

Potter DJ, Clark P, Brown MB (2008) Potency of delta 9-THC and other cannabinoids in cannabis in England in 2005: implications for psychoactivity and pharmacology. Forensic Sci 53(1):90-94

Rathbone J, Variend H, Mehta H (2008) Cannabis and schizophrenia. Cochrane Database Syst Rev 16(3):CD004837

Roser P, Juckel G, Rentzsch J, Nadulski T, Gallinat J, Stadelmann AM (2008) Effects of acute oral Delta9-tetrahydrocannabinol and standardized cannabis extract on the auditory P300 event-related potential in healthy volunteers. Eur Neuropsychopharmacol 18 (8):569-577

Scheurich A, Müller MJ, Wetzel H, Anghelescu I, Klawe C, Ruppe A, Lörch B, Himmerich H, Heidenreich M, Schmid G, Hautzinger M, Szegedi A (2000) Reliability and validity of the German version of the European Addiction Severity Index (EuropASI). J Stud Alcohol 61(6):916-919

Schlicker E, Kathmann M (2001) Modulation of transmitter release via presynaptic cannabinoid receptors. Trends Pharmacol Sci $11: 565-572$

Schwenkreis P, Witscher K, Janssen F, Addo A, Dertwinkel R, Zenz M, Malin JP, Tegenthoff M (1999) Influence of the NMDA antagonist memantine on human motor cortex excitability. Neurosci Lett 270:137-140

Semple DM, McIntosh AM, Lawrie SM (2005) Cannabis as a risk factor for psychosis: systematic review. J Psychopharmacol 19 (2):87-194

Siebner HR, Dressnandt J, Auer C, Conrad B (1998) Continuous intrathecal baclofen infusions induced a marked increase of the transcranially evoked silent period in a patient with generalized dystonia. Muscle Nerve 21:1209-1212

Ujike H, Takaki M, Nakata K, Tanaka Y, Takeda T, Kodama M, Fujiwara Y, Skai A, Kuroda S (2002) CNR1, central cannabinoid receptor gene, associated with susceptibility to hebephrenic schizophrenia. Mol Psychiatry 7:515-518

Voruganti LN, Slomka P, Zabel P, Mattar A, Awad AG (2001) Cannabis induced dopamine release: an in-vivo SPECT study. Psychiatry Res: Neuroimaging Sect 107(3):173-177

Werhahn KJ, Kunesch E, Noachtar S, Benecke R, Classen J (1999) Differential effects on motorcortical inhibition induced by blockade of GABA uptake in humans. J Physiol 517:591-597

Wittchen HU, Wunderlich U, Gruschwitz S, Zaudig M (1997) Strukturiertes Klinisches Interview für DSM-IV. Achse I: Psychische Störungen. A German, advanced adaptation of the original SCID version by RL Spitzer, JB Williams, M Gibbon and MB First. Hogrefe-Verlag, Göttingen 
Wobrock T, Soyka M (2008) Pharmacotherapy of schizophrenia with comorbid substance use disorder - reviewing the evidence and clinical recommendations. Prog Neuropsychopharmacol Biol Psychiatry 32(6):1375-1385

Wobrock T, Sittinger H, Behrendt B, D'Amelio R, Falkai P, Caspari D (2007) Comorbid substance abuse and neurocognitive function in recent-onset schizophrenia. Eur Arch Psychiatry Clin Neurosci 257(4):203-210

Wobrock T, Schneider M, Kadovic D, Schneider-Axmann T, Ecker UK, Retz W, Rösler M, Falkai P (2008) Reduced cortical inhibition in first-episode schizophrenia. Schizophr Res 105(13):252-261
Woods SW (2003) Chlorpromazine equivalent doses for the newer atypical antipsychotics. J Clin Psychiatry 64:663-667

Ziemann U (2004) TMS and drugs. Clinical Neurophysiol 115:1717-1729

Ziemann U, Lönnecker S, Steinhoff BJ, Paulus W (1996a) Effects of antiepileptic drugs on motor cortex excitability in humans: a transcranial magnetic stimulation study. Ann Neuro 40:367-378

Ziemann U, Lönnecker S, Paulus W (1996b) Inhibition of human motor cortex by ethanol. A transcranial magnetic stimulation study. Brain 118(Pt 6):1437-1446

Ziemann U, Chen R, Cohen LG, Hallett M (1998) Dextromethorphan decreases the excitability of the human motor cortex. Neurology $51: 1320-1324$ 